\section{Salud y nutrición del niño indígena chileno (Mapuche)}

\section{The health and nutrition of indigenous children in Chile (Mapuche)}

\section{Saúde e nutrição da criança indígena chilena (Mapuche)}

Hugo Amigo 1

Patricia Bustos 1

doi: 10.1590/0102-311X00073918

\section{Resumen}

El objetivo de este trabajo fue realizar una revisión de la situación de salud y nutrición del niño indígena chileno, específicamente del Mapuche, en lo publicado en las bases de datos de referencias bibliográficas y en investigaciones específicas que tienen base poblacional. Para este trabajo se buscó lo publicado en PubMed, LILACS, organismos nacionales e internacionales, durante los últimos 15 años. Desde el 2006 hasta el 2015 la proporción de pobres era mayor en los indígenas, con disminución de la brecha de 16\% el 2006 a 7,7\% el 2015 ( $p<0,001)$. En la primera década de este siglo la mortalidad infantil en los niños indígenas tenía una tasa de 17,1/1.000 nacidos vivos, mientras en los no indígenas era de de 8,8/1.000y, en el seguimiento de cinco años, la brecha se mantuvo $(p<0,001)$. Los recién nacidos con peso $<2,500 \mathrm{~g}$ el año 2000 no superaban el 6\% (5,6\% en no indígenas y 5,2\% en los indígenas). La talla baja al ingreso a la escuela era 8,4\% en los escolares indígenas y 3,1\% en los no indígenas, disminuyendo a 3,7 en los indígenas y 2,6\% en los no indígenas el 2004, la obesidad en cambio aumentó más en los indígenas, llegando a 24,2y $25,3 \%$ en los no indígenas ( $p<0,001)$. La menarquia se presentó cuatro meses más tarde en las indígenas (12,7 años) y el indice de masa corporal, circunferencia de cintura y el porcentaje de grasa, fueron significativamente mayores en las indígenas en el momento de la telarquia, así como la frecuencia de exceso de peso (55\% vs. $42 \%$ en las no indígenas). Los niños Mapuche presentan una condición de salud y nutrición favorable, comparada con los indígenas de otros países del continente, sin embargo, aun existe una brecha adversa -comparada con los no indígenas-, en que la desigualdad desfavorece al niño indígena y esta debe ser reconocida y corregida.

Niño; Población Indígena; Nutrición

\author{
Correspondencia \\ H. Amigo \\ Departamento de Nutrición, Facultad de Medicina, Universidad \\ de Chile. \\ Independencia 1027, Santiago / Región Metropoitana - \\ 8380453, Chile. \\ hamigo@med.uchile.cl \\ 1 Facultad de Medicina, Universidad de Chile, Santiago, Chile.
}




\section{Introducción}

Los indígenas americanos fueron las primeras personas que habitaron el sur del continente americano hace miles de años. Todavía sigue habiendo comunidades a lo largo y ancho del subcontinente, cada cual con una lengua, costumbres y creencias propias.

El Estado de Chile reconoce como pueblos indígenas a los Aymaras, Quechuas, Atacameños, Collas, Diaguitas, Mapuche, Kawashqar, Alacalufe, Yamana, Yagán y Rapa Nuí 1, siendo los Mapuche los más numerosos (83.8\% de todos los grupos indígenas) 2.

Los Mapuche habitan principalmente en la denominada Región de la Araucanía (centro sur del país), donde se concentra la mayor proporción en pobreza del país y muchos de ellos han migrado a la capital del país (Santiago), aunque también una fracción de ellos habita en la República de Argentina 3. Según la última Encuesta de Caracterización Socioeconómica Nacional (Casen) 4, la cantidad de personas de la etnia indígena, que se identificaron como tal, correspondería a $9 \%$ de la población de 17.574.003 que son los habitantes de Chile en la actualidad 5. Los historiadores indican que eran grupos nómadas, dedicados a la caza y que antes de la llegada de los españoles este grupo alcanzaba una cifra relevante que superaba el $20 \%$ de la población, que fue desapareciendo por aniquilamiento y mestizaje 6 . El idioma de los Mapuche es el mapudungun, sin embargo, cada vez se habla menos, por la influencia del idioma español a través de los distintos medios de comunicación y a pesar del esfuerzo de enseñar este idioma, desde la enseñanza básica, en la zona que ellos habitan. Desde hace algunos años los Mapuche vienen reivindicando aspectos centrales para su desarrollo como: recuperación de sus tierras ancestrales, mayor presencia de políticas públicas que los beneficien y reconocimiento de su identidad cultural.

Los indígenas de América Latina tienen un patrón de edad más joven que el resto de la población, observándose que la pirámide poblacional de los grupos indígenas tiene una base más ancha y una cúspide más pequeña que la de los no indígenas 7 . En Chile no hay mayores diferencias en estas proporciones, observándose que la población indígena menor de 18 años, es algo mayor que la no indígena con cifras de 18,1 y $17,7 \%$ respectivamente 7 .

Históricamente, los indígenas del continente han sido marginalizados del desarrollo socioeconómico, constituyéndose en los grupos de mayor pobreza, lo que se ha reflejado en que los indicadores de calidad de vida y salud sean más desfavorables en ellos 8 .

El objetivo de este trabajo fue hacer una revisión de la situación de salud del niño indígena chileno, específicamente del Mapuche, en lo publicado en las bases de datos de referencias bibliográficas y en investigaciones específicas de población indígena mapuche que tienen base poblacional.

\section{Material y métodos}

Para esta revisión se accedió a lo publicado, desde el inicio de la primera década de este milenio, utilizando las siguientes palabras claves: "mortalidad en la infancia”, "estado de salud” y "nutrición en indígenas chilenos de origen mapuche”. Para ello, se utilizaron las bases de datos PubMed, LILACS, y los resultados de investigaciones específicas relacionados con el área temática, además de los censos poblacionales, encuestas nacionales y publicaciones oficiales de organismos del sistema de Naciones Unidas que hayan considerado a los indígenas que habitan en Chile, estas búsquedas se realizaron en idioma español, portugués e inglés. La búsqueda inicial incluyó 38 estudios, en los que se logró identificar y agrupar información sobre condicionantes socioeconómicos, mortalidad infantil, situación de los recién nacidos, problemas nutricionales por déficit y exceso de peso. Para el análisis del estado nutricional se utilizó el patrón de crecimiento sugerido por el Centro Nacional de Estadísticas en Salud de los Centros de Control y Prevención de Enfermedades de los EE.UU. (NCHS/CDC) 9 .

Los resultados que se presentan fueron obtenidos de la bibliografía consultada y cuando fue necesario se realizaron análisis complementarios. Debido a que gran parte de la información es producto de investigaciones efectuadas por el grupo que presenta este trabajo, se compararon promedios de peso y talla al nacer entre indígenas y no indígenas, utilizando análisis de varianza (ANOVA). En el caso de las Figuras 1 y 2, las diferencias de proporciones entre indígenas y no indígenas, en los distin- 
tos años o según tasas de mortalidad, se efectuaron mediante la prueba de chi cuadrado. Para evaluar la interacción entre etnia y tiempo se usó regresión logística.

Los trabajos realizados por nuestro grupo de investigación, y analizados en este trabajo, contaron con la aprobación del Comité de Ética de la Facultad de Medicina de la Universidad de Chile, quien se adhiere a la Declaración de Helsinki. Los padres de los niños participantes firmaron un consentimiento informado, previo al ingreso en el estudio y los mayores de 12 años firmaron un asentimiento informado.

\section{Resultados}

\section{Situación socioeconómica}

Para entender la situación de salud de los niños indígenas chilenos es relevante analizar el contexto socioeconómico en el que les ha correspondido crecer y desarrollarse. Hay dos fuentes que son claves para analizar esta condición, los censos de población y las encuestas Casen que se han realizado estas últimas décadas, con intervalos de dos a tres años 4 .

Las encuestas Casen analizan diferentes formas de medición de la situación socioeconómica, una de ellas es medir la pobreza a través de los ingresos. De acuerdo con esto, desde el año 2006 hasta el 2015, hay una disminución de la pobreza e indigencia (pobreza extrema), tanto en indígenas, como no indígenas, la proporción de población calificada de pobre es mayor en los indígenas, verificándose una disminución de la brecha de pobreza de 16 puntos porcentuales el 2006, hasta 7,7\% el año 2015, diferencia que es significativa en todos los años analizados (Figura 1) 4.

\section{Figura 1}

Porcentaje en situación de pobreza según pertenencia a pueblos indígenas, Chile, 2006-2015.

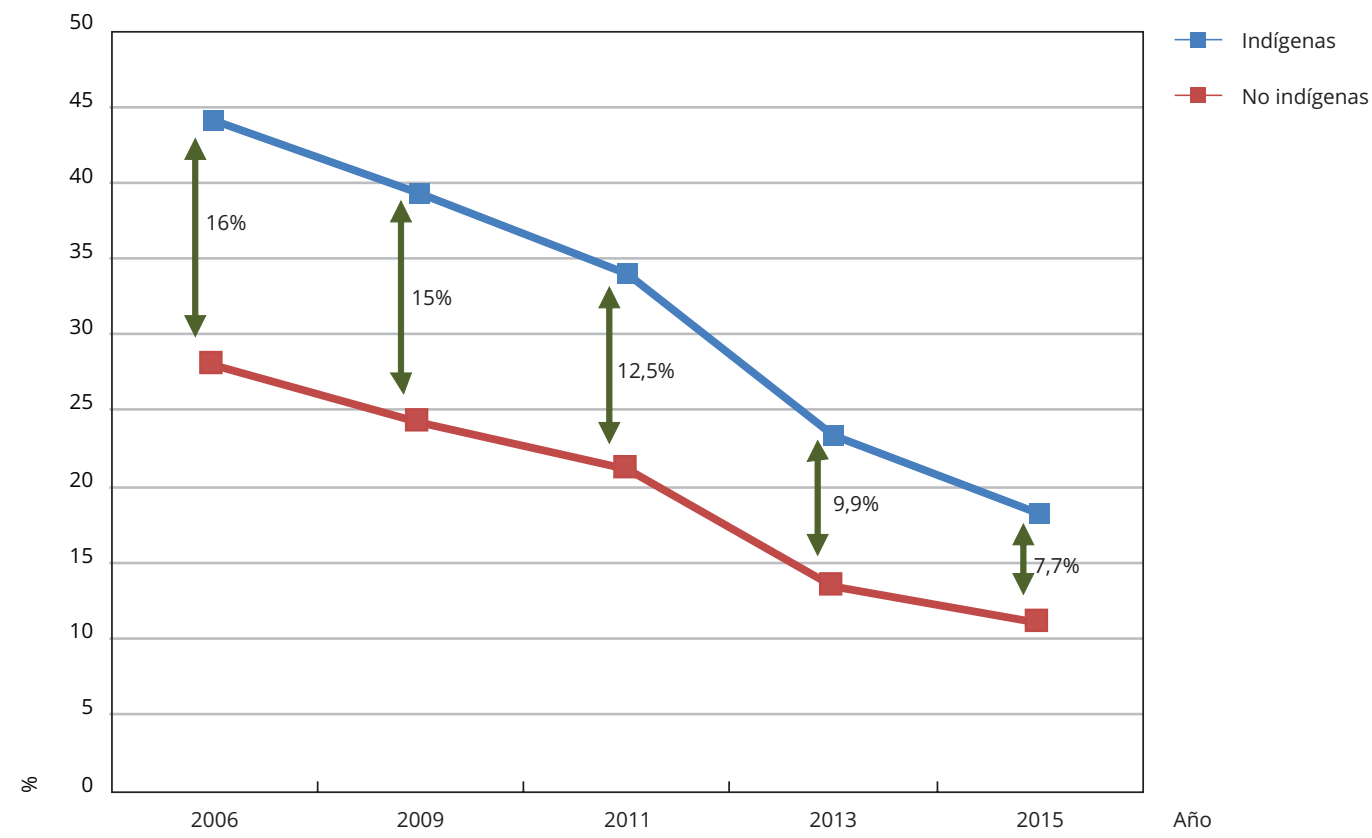

Fuente: Ministerio de Desarrollo Social de Chile. 


\section{Mortalidad infantil}

La mortalidad infantil es un indicador que se ha utilizado para señalar el grado de desarrollo de los países, especialmente en los grupos de riesgo social, uno de estos grupos son los indígenas del continente, especialmente los que habitan en áreas rurales 10,11. La reducción de la mortalidad infantil continúa siendo uno de los objetivos en salud en los países y esta reducción constituye parte de las estrategias para disminuir la pobreza.

En América Latina ha habido una disminución del número de muertes en el primer año de vida, aunque en muchos lugares persisten cifras inaceptables, observándose que en los países más pobres hay mayores índices de mortalidad en la infancia ${ }^{12}$. Chile se ha clasificado como un país de nivel de desarrollo intermedio, que presenta tasas satisfactorias de este indicador, sin embargo, aún hay grupos que presentan una situación más desventajosa, revelando una situación de inequidad en ese país 13 .

Desafortunadamente, en Chile las estadísticas vitales no divulgan información desagregada por etnia indígena por falta de un identificador poblacional, a pesar que ya hace algunos años se encuentra disponible en el país un listado de apellidos de origen Mapuche con más de 7.000 de esta procedencia 14 , que podría servir de base para identificar las personas de esta etnia.

Entre los datos disponibles figura un estudio que aborda las tasas de mortalidad infantil, de acuerdo a la información del censo del año 2002, donde se señala que para la etnia Mapuche alcanzaba a 12,8 por mil nacidos vivos, mientras que en el resto de la población llegaba a 11,6, lo que viene a indicar una brecha de 1,1 10. Posteriormente, se informa que en el año 2015 la mortalidad infantil en Chile continúa disminuyendo, llegando a un valor de 6,8 por mil nacidos vivos 15 .

En un estudio específico de mortalidad en la infancia, llevado a cabo con datos nacionales entre el año 2000 a 2004, en que sí se consideró la procedencia de los niños fallecidos, a través de los apellidos, se dividieron las muertes entre los que no tenían ningún, uno o dos apellidos indígenas (los de ambos padres), observándose que la mortalidad infantil para el conjunto de los años estudiados alcanzó una tasa de 9/1.000 nacidos vivos, la tasa de los fallecidos con dos apellidos indígenas era de 17,1, mientras que la de los no indígenas alcanzaba a 8,8. Las tasas de mortalidad neonatal que corresponden a un $62 \%$ de las muertes ocurridas en el primer año de vida, y de éstas, un 76\% ocurren dentro de la primera semana. Al calcular las tasas de mortalidad perinatal, neonatal y post neonatal por etnia, se comprobó que en todas ellas la mortalidad en los niños con dos apellidos Mapuche superan a las de los niños sin apellidos de esta procedencia, así, en el caso de la mortalidad perinatal, las diferencias de tasas fueron de 2,5, en la neonatal 3,9, y en la postneonatal 4,5 veces mayores, comparadas con las observadas en los niños sin apellidos mapuche que fallecen (Figura 2) 16.

En el análisis por año, se observó que las tasas de mortalidad infantil de los niños sin o con un apellido Mapuche experimentan menores cambios a través de los años, mientras que en los con dos apellidos Mapuche son más altas, pero también son las que más disminuyen (Figura 3).

\section{Peso al nacer}

Esta medición en el recién nacido refleja las condiciones de salud y nutrición de la madre durante el embarazo, sumado a factores medio ambientales y genéticos, por esta razón constituye un buen indicador que permite explicar, en parte, el crecimiento del niño durante los primeros meses de vida y el efecto a largo plazo en numerosas enfermedades crónicas 17.

Como se señaló previamente, las informaciones sobre la situación nutricional a nivel nacional no están desagregadas por etnia, pero se dispone de información que indirectamente nos informa de la situación nutricional de la población indígena, al localizarse sus residencias principalmente en la Región de La Araucanía. También hemos podido tener una aproximación al problema, a través de estudios específicos, información que se presenta a continuación.

En un estudio realizado al inicio de la primera década de este milenio, considerando los nacidos vivos en el país entre los años 2000 a 2004 ( $n=1.166 .513)$, donde se identificaron los apellidos de procedencia Mapuche, se constató que el peso al nacer se mantuvo en valores satisfactorios a través de los años estudiados, con valores promedio levemente superiores en los recién nacidos de procedencia indígena, lo mismo ocurrió con la longitud al nacer (Tabla 1). El porcentaje de recién nacidos con 


\section{Figura 2}

Tasas de mortalidad infantil, perinatal, neonatal y postneontal según apellidos de origen indígena Mapuche. Chile, 2000-2004.

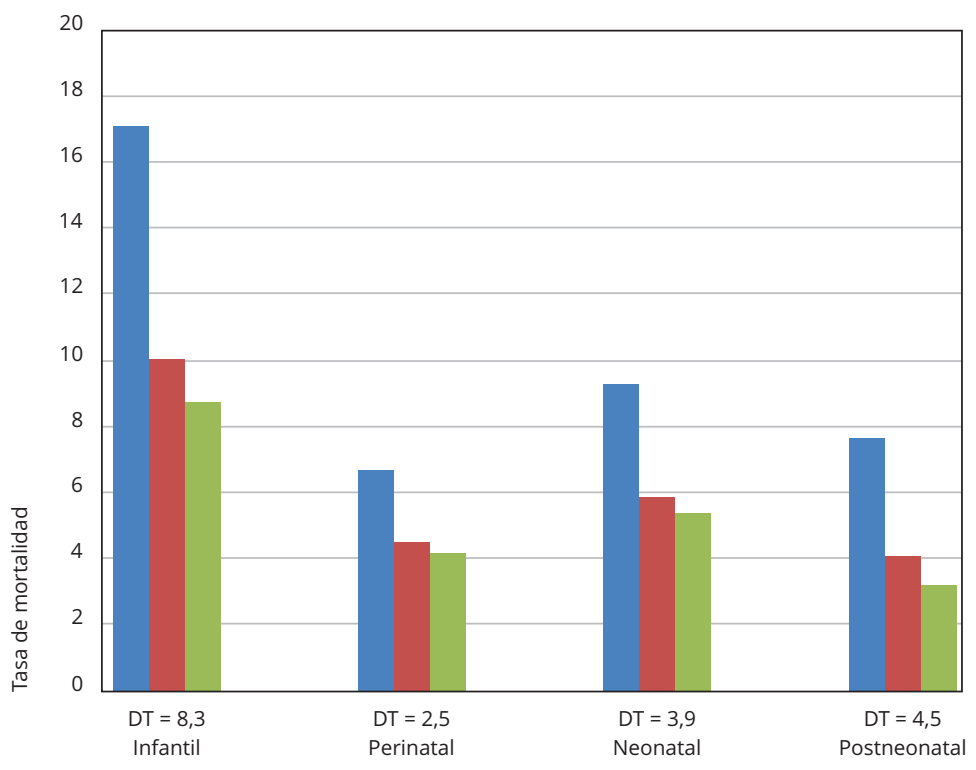

DT: diferencia de tasas entre niños con dos apellidos vs. sin apellidos indígenas.

Fuente: Bustos \& Amigo 16.

\section{Figura 3}

Tasas de mortalidad infantil (TMI) según grupo étnico (número de apellidos Mapuche). Chile, 2000-2004.

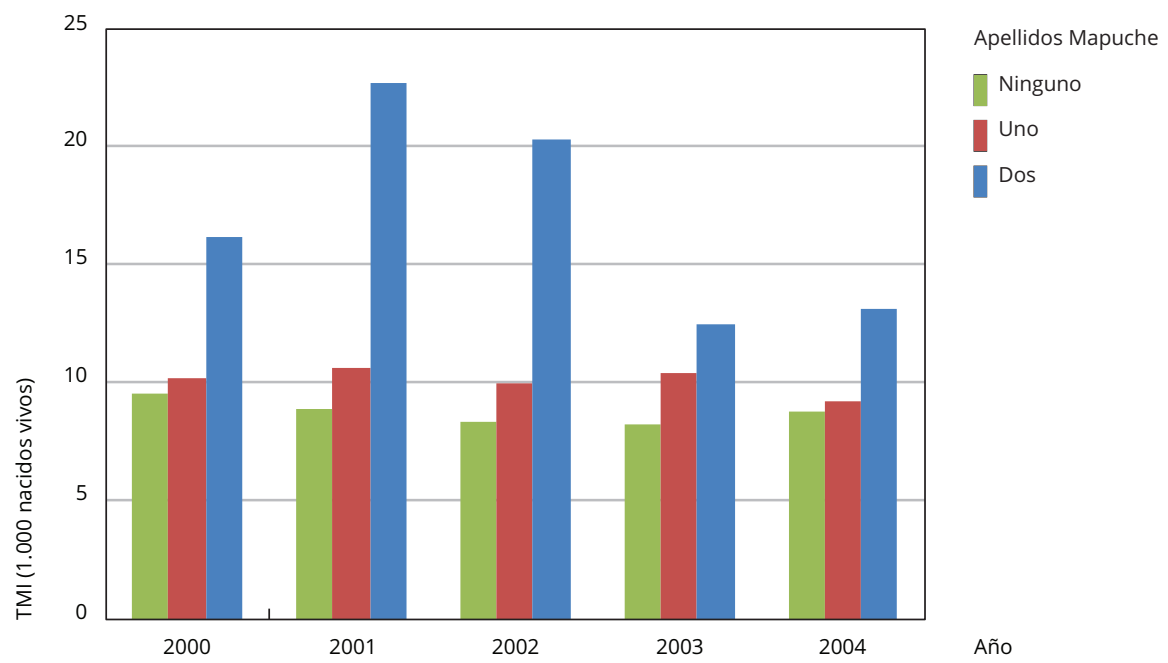

DT: diferencia de tasas entre niños con dos apellidos vs. sin apellidos indígenas.

Fuente: Bustos \& Amigo 16 . 
Tabla 1

Promedio del peso y longitud al nacer de recién nacidos de procedencia Mapuche y no Mapuche entre los años 2000 y 2004, Chile.

\begin{tabular}{|c|c|c|c|c|}
\hline & \multicolumn{2}{|c|}{ Peso de nacimiento (g) } & \multicolumn{2}{|c|}{ Longitud al nacer $(\mathrm{cm})$} \\
\hline & $X(D E)$ & Valor de $p$ & $X(D E)$ & Valor de $p$ \\
\hline 2000 & & 0,0001 & & 0,028 \\
\hline Mapuche $(n=16.726)$ & $3.379(604)$ & & $49,7(3,78)$ & \\
\hline No Mapuche $(n=221.184)$ & $3.352(572)$ & & $49,6(3,6)$ & \\
\hline 2001 & & 0,0001 & & 0,0001 \\
\hline Mapuche $(n=17.043)$ & $3.344(857)$ & & $48,8(8,5)$ & \\
\hline No Mapuche $(n=220.060)$ & $3.290(881)$ & & $48,5(9,2)$ & \\
\hline 2002 & & 0,0001 & & 0,032 \\
\hline Mapuche $(n=14.594)$ & $3.389(623)$ & & $49,5(3,4)$ & \\
\hline No Mapuche $(n=216.531)$ & $3.353(616)$ & & $49,5(3,8)$ & \\
\hline 2002 & & 0,0001 & & 0,032 \\
\hline Mapuche $(n=15.030)$ & $3.380(619)$ & & $49,6(3,5)$ & \\
\hline No Mapuche $(n=224.993)$ & $3.343(586)$ & & $49,5(3,4)$ & \\
\hline 2004 & & 0,0001 & & 0,0001 \\
\hline Mapuche $(n=15.273)$ & $3.402(694)$ & & $49,7(4,4)$ & \\
\hline No Mapuche ( $n=215.079$ ) & $3.347(618)$ & & $49,5(3,7)$ & \\
\hline
\end{tabular}

Nota: diferencias entre Mapuche y No Mapuche se estimaron mediante análisis de varianza (ANOVA).

bajo peso $(<2.500 \mathrm{~g})$ no supera el $6 \%$ (5,6\% en los no indígenas y $5,2 \%$ en los indígenas), mientras que el porcentaje de recién nacidos con peso de nacimiento $>4.000 \mathrm{~g}$ fue 9,1 y 10,8\%, respectivamente 18 .

\section{Problemas nutricionales por déficit}

En la actualidad, la desnutrición infantil y del pre-escolar es de baja magnitud en el país. En los menores de 6 años, según la información del Ministerio de Salud de Chile, alcanza a un 0,3\% y estas cifras se han mantenido desde hace varios años. La Región de la Araucanía tiene cifras similares (0,3\%).

En los escolares que ingresan a la escuela primaria, según informes del Ministerio de Educación en el año 2015, la prevalencia de desnutrición era 2,1\% a nivel nacional, siendo aún menor en la Región de La Araucanía (1,4\%), con diferencias según área geográfica, ya que las cifras en esta Región son $1,2 \%$ para los escolares urbanos, y el doble para los rurales, zonas donde se concentra la población más pobre e indígena del país. El retraso de talla según esta misma fuente alcanzó en estos escolares cifras de 2,9\%, levemente menores en la Región de la Araucanía (2,2\%).

En un estudio realizado, con información proporcionada por el Ministerio de Educación de Chile, en niños pertenecientes a escuelas públicas, que ingresaron a primer año básico entre los años 1997 a 2005 ( $\mathrm{n}=1.757 .155)$, se constató que aquellos ubicados < -2 puntajes $\mathrm{z}$ del índice de masa corporal (IMC) no superaban el 2\%, y no fue más frecuente en los niños de procedencia Mapuche 19. En este mismo estudio, la talla baja (<-2 DE de talla para la edad) fue más frecuente en los niños de procedencia Mapuche, observándose que al inicio de la serie el 8,4\% en los escolares con 2 apellidos Mapuche, $4,8 \%$ en los con uno y 3,1\% en los con apellidos chileno-español. Esta condición disminuyó en los tres grupos a valores bajo 4 al final de la serie analizada 19 .

La información expuesta da cuenta de las mejorías ocurridas en el déficit de peso o talla de la población escolar, ya que estudios previos reflejaban déficit de tallas mayores, las que estaban diferenciadas por nivel socioeconómico. En comunas de extrema pobreza los niños de procedencia mapuche presentaban promedios de puntajes z - 1.1 mientras los no indígenas en las mismas comunas era -0,70. En comunas de mediana vulnerabilidad estos valores eran -0,5, tanto para indígenas y no indígenas, para mejorar a -0,21 en los indígenas de baja vulnerabilidad, comparados con -0,37 de los no indígenas (tendencia significativa en ambos grupos, p < 0,001) (Figura 4) 20. 


\section{Figura 4}

Puntaje z de talla para la edad em niños indígenas y no indígenas según vulnerabilidad social.

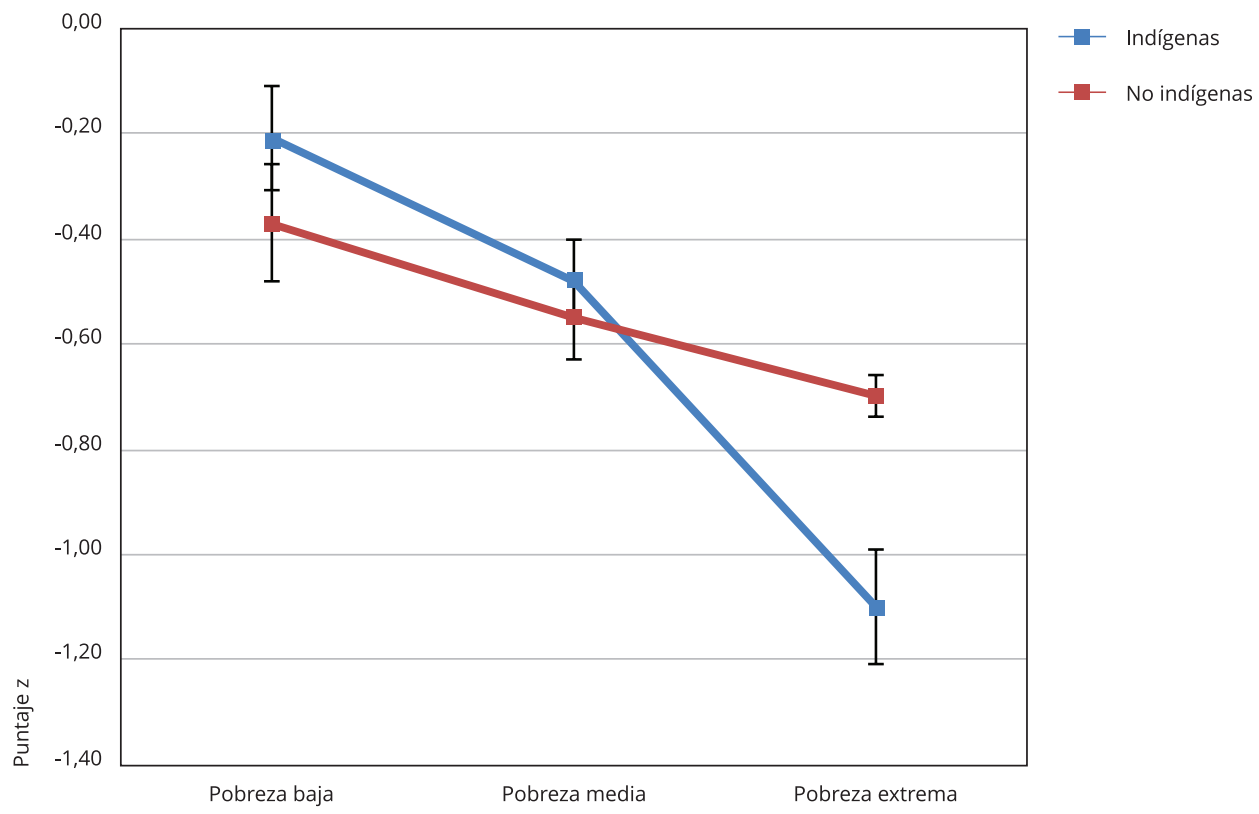

Fuente: Bustos et al. 20

\section{Problemas nutricionales por exceso de peso}

Si bien la condición de déficit de peso y talla son poco frecuentes, el exceso de peso alcanza en la actualidad cifras alarmantes y en incremento en el país, con 10,3\% de obesidad en los menores de 6 años, cifra que es algo mayor en los niños que viven en la Región de La Araucanía (12,1\%).

En los escolares de primer año básico, las cifras son más del doble que las reportadas en menores de 6 años, a nivel nacional, e incluso mayores en las reportadas en población de la Región de la Araucanía (24,2 y 25,3\%, respectivamente).

En un estudio realizado en escolares de igual edad, donde se identificó la procedencia étnica según número de apellidos, se constató una situación inversa, ya que los que tenían menos obesidad al inicio de la serie eran los Mapuche con 2 apellidos, cifras que fueron incrementando en una tasa de 5,4\%, comparadas con los que tenían un apellido o tenían apellidos chileno-español, que incrementaron sólo en 4,1\%, llegando en el 2005 a cifras similares en los 3 grupos estudiados (entre 20 y 22\%) (Figura 5) 19.

En vista de la situación homogénea entre In y Nin de déficit de talla, desde el nacimiento hasta el ingreso a la escuela, fue de interés conocer el crecimiento de los adolescentes, ya que nuevamente tienen aceleración de la velocidad de crecimiento y es un periodo que está influenciado por factores tanto genéticos como ambientales.

En un estudio de seguimiento de dos cohortes de niñas indígenas (Mapuche) y no indígenas, en que se evaluó la aparición de eventos puberales y su crecimiento, se comprobó que la telarquia en la Región de La Araucanía se presenta a los 10 años y 4 meses, se adelanta con el exceso de peso, se atrasa con el déficit de talla y no se encuentra modificada por la etnia 21. También se comprobó que el IMC, la circunferencia de cintura y la masa grasa fueron significativamente mayores a esta edad en las indígenas 22 .

La menarquia se presentó 4 meses más tarde en las niñas de procedencia indígena (12 años 7 meses vs. 12 años 3 meses en las no indígenas), y en cuanto a las medidas antropométricas se constató que 
Figura 5

Evolución de la prevalencia de obesidad según origen étnico.

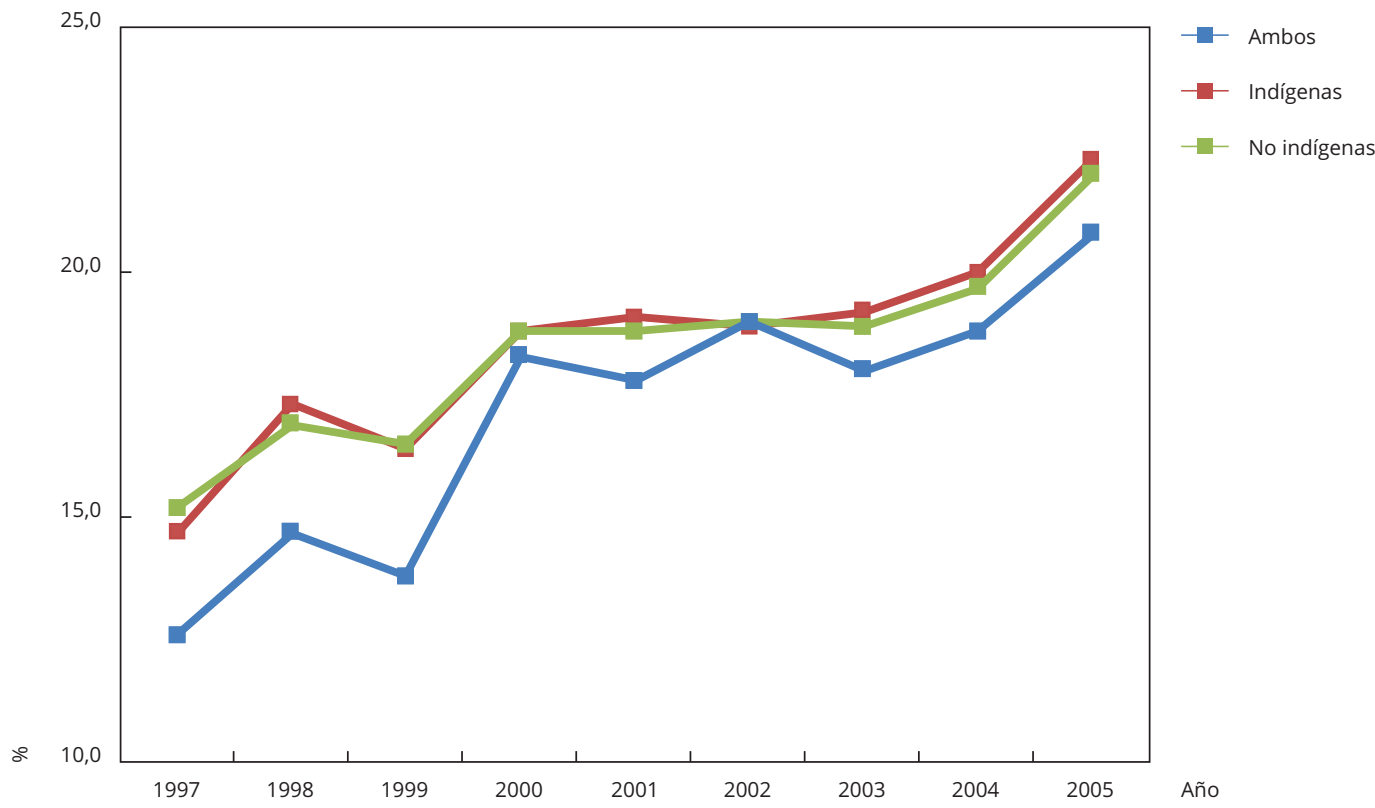

Fuente: Bustos et al. 20

tanto el IMC, la circunferencia de cintura y el porcentaje de grasa fueron mayores en las niñas indígenas, así como la frecuencia de exceso de peso (55\% vs. $42 \%$, respectivamente; $\mathrm{p}<0,001) 23$. Respecto al crecimiento en estatura de estas cohortes, se comprobó que las niñas indígenas eran más bajas que las no indígenas en el momento de la menarquia, y que el aumento de estatura durante los 36 meses post menarquia fue también menor en las niñas indígenas. El crecimiento en estatura de las niñas estudiadas era inferior al esperado por referencia internacional (ambos grupos étnicos), lo que hace suponer que también lo será su estatura final 24.

\section{Discusión}

Los niños indígenas chilenos (de origen Mapuche) reflejan una condición positiva comparada con la de los indígenas de otros países del continente 25,26,27. No obstante, en la mayoría de los indicadores analizados, los niños indígenas presentan una condición desfavorable al compararlos con los no indígenas, lo que refleja que los avances socioeconómicos han llegado a diferentes grupos poblacionales, incluida la población indígena chilena, pero por otra parte se comprueba una brecha de desigualdad, que desfavorece al niño indígena y esto debe ser reconocido y corregido.

Entre las áreas temáticas que merecen ser destacadas, están los indicadores del estado nutricional en que han ocurrido relevantes avances, uno de ellos es el peso al nacer, en que la mayoría de la población alcanza la recomendación de la Organización Mundial de la Salud (OMS) para niños de término, y esto es tanto para la población indígena, como la no indígena ${ }^{9}$. El otro indicador que merece ser mencionado es el déficit de crecimiento (talla para la edad), que también se acerca a la distribución poblacional sugerida internacionalmente, comprobándose que los niños indígenas que viven en buenas condiciones sociales crecen igual que los no indígenas 20 , lo que refleja que el déficit de crecimiento observado en diferentes lugares es independiente de la procedencia étnica hasta el 
ingreso a la escuela. Esta situación es más favorable que la descrita para otros países de América Latina y refleja el éxito de los programas implementados en el país (focalizándose en la población de menores recursos, incluida la indígena), que han contribuido a la prevención de la desnutrición en los primeros años de la vida, programas que se extienden a la edad preescolar y escolar. También sugiere que no es necesario utilizar un patrón de referencia diferente para las poblaciones indígenas en los menores de 6 años, ya que si esta población crece, y se desarrolla en un ambiente favorable, puede alcanzar todo su potencial de crecimiento.

En relación a la obesidad, se comprueba el aumento progresivo en los grupos estudiados, principalmente en los niños con dos apellidos mapuche, esto reflejaría el cambio de sus hábitos alimentarios y la menor actividad física que realizan en actividades diarias (caminar, trabajo, deportes). Si sigue esta tendencia y no se interviene se puede aventurar que este problema será más frecuente en los años próximos, especialmente en la población mapuche, y esto es una alerta para evitar consecuencias negativas, a las que se asocia el exceso de peso.

Sin duda uno de los problemas que enfrentamos en esta revisión es que en las estadísticas vitales no hay información de la situación de las personas de la etnia indígena, y mucho menos a qué grupo étnico pertenecen, lo que es una limitación, porque las estadísticas relacionadas con registros de carácter continuo (defunciones, nacimientos, matrimonios acuerdo civiles, separaciones divorcios, adopciones), no están actualizados por etnia. El no disponer de esta información obliga a realizar estudios específicos, donde se pueda identificar adecuadamente a la población de procedencia indígena, pero, lamentablemente, éstos rápidamente quedan desactualizados.

Se han descrito desigualdades en los indicadores básicos que miden la situación de salud y nutrición en indígenas, comparados con los no indígenas, sin embargo, los marcadores de etnia en distintos lugares son diferentes. En algunos países, se ha señalado que se denomina indígena a quienes viven en un lugar compartiendo territorios y ancestros, pero en muchos lugares esto no se cumple, ya que las personas que viven en una misma comunidad no tienen esa condición, la otra definición es la que comparten costumbres e idioma 28. Esto, que es posible en algunos lugares del continente, no es posible hacerlo con los mapuche, donde hay una paulatina pérdida del idioma de una generación a otra, como lo ha documentado recientemente el Ministerio de Desarrollo Social de Chile 4. Debido a lo anterior, se ha realizado un catastro de apellidos indígenas de origen mapuche que se espera sea utilizado 14 .

En cuanto a los problemas nutricionales al ingreso a la escuela, el haber encontrado frecuencias mayores de talla baja en escolares mapuche al comienzo de la serie estudiada (especialmente, en los con dos apellidos de esta procedencia) y que disminuyen al avanzar los años indica una tendencia favorable de este problema nutricional y también la deseable reducción de las diferencias entre etnias.

Los antecedentes expuestos en este manuscrito señalan que hay avances positivos de la situación de los niños indígenas de origen Mapuche en Chile, en comparación con los indígenas de otros lugares de América Latina. No obstante, continúan persistiendo las diferencias desfavorables con la población no indígena que habita en el país, ante lo cual se debe continuar con acciones prioritarias y focalizadas, considerando aspectos socioeconómicos y políticos, aunque respetando su cultura y ancestros. El haber encontrado una desviación hacia el exceso de peso en los niños indígenas, que es mayor que en el resto de la población, debe ser una alerta para evitar consecuencias adversas a medio y largo plazo. También, estos resultados destacan la necesidad de contar con información de los indígenas de forma periódica y actualizada entre la que se debe mencionar las estadísticas vitales y los indicadores que miden el desarrollo. 


\section{Colaboradores}

H. Amigo y P. Bustos contribuyeron con la redacción del manuscrito, análisis de información y revisión final.

\section{Informaciones adicionales}

ORCID: Hugo Amigo (0000-0002-3301-5749); Patricia Bustos (0000-0001-8674-6142).

\section{Agradecimientos}

Parte de la información expuesta en esta publicación, proviene de proyectos financiados por el Fondo de Investigación en Salud del Estado Chileno (FONIS), proyecto SA05i20077 y del Fondo de Investigación en Ciencia y Tecnología de Chile (FONDECYT), proyecto ${ }^{\circ} 1060884$.

\section{Referencias}

1. Gobierno de Chile. Ley indígena no 19.253. http://www.leychile.cl (accedido el 03/ Abr/2018).

2. Ministerio de Desarrollo Social. Casen 2015. Pueblos indígenas. Síntesis de resultados. http://observatorio.ministeriodesarrollosocial.gob.cl/casen-multidimensional/casen/ docs/CASEN_2015_Resultados_pueblos_indigenas.pdf (accedido el 10/Abr/2018).

3. Martínez C, Barragán A. Mapuches del Neuquén. Arte y cultura en la Patagonia Argentina. Buenos Aires: Luz Editora; 2002.

4. Ministerio de Desarrollo Social. Casen 2015 Ampliando la mirada sobre la pobreza y la desigualdad. http://observatorio.ministeriodesarrollosocial.gob.cl/casen-multidimensional/ casen/docs/CASEN_2015_Ampliando_la_mi rada_sobre_la_pobreza_desigualdad.pdf (accedido el 10/Abr/2018).

5. Instituto Nacional de Estadísticas. Censo 2002. Síntesis de resultados. http://www.ine. $\mathrm{cl} /$ docs/default-source/FAQ/s\%C3\%ADntesisde-resultados-censo-2002.pdf?sfvrsn=2 (accedido el 10/Abr/2018).

6. Bengoa J. Historia del pueblo Mapuche: (siglo XIX y XX). Santiago: Editorial Interamericana Ltda.; 1996.

7. Naciones Unidas; Comisión Económica para América Latina; Organización Panamericana de la Salud. Salud de la población joven indígena en América Latina: un panorama general. Santiago: Naciones Unidas; 2011.

8. Giuffrida A. Racial and ethnic disparities in health in Latin America and the Caribbean. New York: Inter-American Development Bank; 2007.

9. Centers for Disease Control and Prevention. Growth charts for the United States: methods and development. http://www.cdc.gov/nchs/ data/sr11_246.pdf (accedido el 10/Abr/2018).

10. Comisión Económica para América Latina. Mortalidad infantil y en la niñez de pueblos indígenas y afrodescendientes de América Latina: inequidades estructurales, patrones diversos y evidencia de derechos no cumplidos. Santiago: Comisión Económica para América Latina; 2010.

11. Del Popolo F, Oyarce A. Población indígena de América Latina: perfil sociodemográfico en el marco de la Conferencia Internacional sobre Población y Desarrollo y de las metas del Milenio. Notas de Población 2005; (79):13-52.

12. Fondo de las Naciones Unidas para la Infancia. Estado Mundial de la Infancia 2016. Una oportunidad para cada niño. https://www.unicef org/spanish/publications/files/UNICEF_ SOWC_2016_Spanish.pdf (accedido el 10/ Abr/2018).

13. Hertel-Fernandez AW, Giusti AE, Sotelo JM The Chilean infant mortality decline: improvement for whom? Socioeconomic and geographic inequalities in infant mortality, 1990-2005. Bull World Health Organ 2007; 85:798-804. 
14. Amigo H Bustos P. Apellidos mapuche: historia y significado. Santiago: Impresos Maigret Ltda.; 2008.

15. Ministerio de Desarrollo Social. Casen 2011. Encuesta de Caracterización Socioeconómica Nacional. http://observatorio.ministeriodesa rrollosocial.gob.cl/layout/doc/casen/pobre za_casen_2011.pdf (accedido el 10/Abr/2018).

16. Bustos P, Amigo H. Salud materno infantil de la etnia mapuche (mortalidad y estado nutricional). Santiago: Fondo Nacional de Investigación en Salud; 2006.

17. Barker D. Mothers, babies and health in later life. London: Churchill Livingstone; 1998.

18. Amigo H, Bustos P, Kaufman JS. Absence of disparities in anthropometric measures among Chilean indigenous and non-indigenous newborns. BMC Public Health 2010; 10:392.

19. Bustos P, Munoz S, Vargas C, Amigo H. Evolution of the nutritional situation of indigenous and non-indigenous Chilean schoolchildren. Ann Hum Biol 2009; 36:298-307.

20. Bustos P, Amigo H, Munoz SR, Martorell R. Growth in indigenous and nonindigenous Chilean schoolchildren from 3 poverty strata. Am J Public Health 2001; 91:1645-9.

21. Bustos P, Amigo H, Muzzo S, Ossa X. Thelarche and nutritional status: an epidemiological study of two ethnic groups. Rev Med Chile 2009; 137:1301-8.

22. Amigo H, Costa Machado T, Bustos P. Estado nutricional en la telarquia y menarquia de adolescentes chilenas indígenas y no indígenas. Arch Latinoam Nutr 2009; 59:260-5.
23. Amigo H, Lara M, Bustos P, Munoz S. Postmenarche growth: cohort study among indigenous and non-indigenous Chilean adolescents. BMC Public Health 2015; 15:51.

24. Lara M, Bustos P, Amigo H. Change in postmenarche anthropometric indicators in indigenous and nonindigenous adolescents from Chile. Am J Hum Biol 2017; 29:e23006.

25. Ferreira AA, Welch JR, Cunha GM, Coimbra Jr. CE. Physical growth curves of indigenous Xavante children in Central Brazil: results from a longitudinal study (2009-2012). Ann Hum Biol 2016; 43:293-303.

26. Coimbra Jr. CE, Santos RV, Welch JR, Cardoso AM, de Souza MC, Garnelo L, et al. The First National Survey of Indigenous People's Health and Nutrition in Brazil: rationale, methodology, and overview of results. BMC Public Health 2013; 13:52.

27. Freire WB, Silva-Jaramillo KM, RamirezLuzuriaga MJ, Belmont P, Waters WF. The double burden of undernutrition and excess body weight in Ecuador. Am J Clin Nutr 2014; 100:1636S-43.

28. Montenegro RA, Stephens C. Indigenous health in Latin America and the Caribbean. Lancet 2006; 367:1859-69. 


\section{Abstract}

The aim of this study was to conduct a review of the health and nutritional status of Chilean indigenous children, specifically Mapuche children, as published in the literature and specific population-based studies. The searches were conducted in PubMed and LILACS in the last 15 years. From 2006 to 2015, the poverty rate was higher in the indigenous population, with a decrease in the gap from 16\% in 2006 to $7.7 \%$ in 2015 ( $p<0.001)$. In the first decade of this century, infant mortality in indigenous children was 17.1/1,000 live births, while in non-indigenous children it was 8.8/1,000, and the gap was maintained in the five-year follow-up $(p<0.001)$. Newborns with birthweight < 2,500g in the year 2000 did not reach 6\% (5.6\% in non-indigenous and 5.2\% in indigenous children). Low height at first school enrollment was 8.4\% in indigenous schoolchildren and 3.1\% in non-indigenous children, decreasing to $3.7 \%$ in indigenous children and $2.6 \%$ in non-indigenous children in 2004, while obesity increased more in indigenous children, reaching $24.2 \%$ in indigenous and $25.3 \%$ in non-indigenous children $(p<0.001)$. Menarche appeared four months later on average in indigenous girls (12.7 years), and body mass index, waist circumference, and fat mass were significantly greater in indigenous girls at the time of thelarche, as was the overweight rate (55\%,vs. 42\% in non-indigenous). Mapuche children show favorable health and nutritional status compared to indigenous children elsewhere in Latin America, but there is still an adverse gap compared to non-indigenous Chilean children. This inequality affecting indigenous Chilean children should be acknowledged and corrected.

Child; Indigenous Population; Nutrition

\section{Resumo}

O objetivo deste trabalho foi realizar una revisão da situação da saúde e nutrição da criança indígena chilena, especificamente da Mapuche, nas publicações relacionadas em bases de dados de referências bibliográficas e em pesquisas específicas que têm base populacional. A pesquisa foi realizada em publicações relacionadas: PubMed, LILACS, organismos nacionais e internacionais, nos últimos 15 anos. Desde 2006 até 2015 a proporção de pobres era maior nos indígenas, com diminuição de uma diferença de 16\% em 2006 a 7,7\% em 2015 ( $p<0.001)$. Na primeira década de este século a mortalidade infantil nas crianças indígenas teve una taxa de 17,1/1.000 nascidas vivas, enquanto nos não indígenas era de 8,8/1.000 $e$, em um acompanhamento de cinco anos, a diferença se manteve $(p<0,001)$. Os recém-nascidos com peso < 2.500g no ano de 2000 não superavam $6 \%$ (5,6\% em não indígenas e 5,2\% nos indígenas). A baixa estatura ao momento do ingresso na escola era $8,4 \%$ nos escolares indígenas e 3,1\% nos não indígenas, diminuindo a 3,7 nos indígenas e 2,6\% nos não indígenas em 2004, a obesidade pelo contrário teve um aumento maior nos indígenas, chegando a 24,2 e 25,3\% nos não indígenas ( $p<$ 0,001). A menarca ocorreu quatro meses mais tarde nas indigenas (12,7 anos) e o indice de massa corporal, circunferência da cintura e a porcentagem da gordura, foram significativamente maiores nas indígenas no periodo da telarca, à semelhança da frequência do sobrepeso (55\% vs. $42 \%$ nas não indígenas). As crianças Mapuche apresentam uma condição de saúde e nutrição favorável, comparada com os indígenas de outros países do continente, no entanto, ainda existe uma brecha adversa comparada com os não indígenas - , onde a desigualdade desfavorece a criança indígena e por isso deve ser reconhecida e corrigida.

Criança; População Indígena; Nutrição
Recibido el 12/Abr/2018

Versión final presentada el 28/Feb/2019 Aprobado el 25/Mar/2019 